\title{
Effect of shot peening parameters to residual stress profiles and Barkhausen noise
}

\author{
Aki Sorsa ${ }^{\mathrm{a}}$, Suvi Santa-aho, ${ }^{\mathrm{b},}$, Jukka Wartiainen ${ }^{\mathrm{c}}$, Lasse Suominen ${ }^{\mathrm{c}}$, Minnamari Vippola ${ }^{\mathrm{b}}$, Kauko \\ Leiviskäa \\ ${ }^{a}$ University of Oulu, Control Engineering, P.O. Box 4300, University of Oulu, Finland \\ b Tampere University of Technology, Laboratory of Materials Science, P.O. Box 589, 33101 \\ Tampere, Finland \\ ${ }^{\mathrm{c}}$ Stresstech Oy, Tikkutehtaantie 1, 40800 Vaajakoski, Finland \\ *corresponding author: Suvi Santa-aho, suvi.santa-aho@tut.fi, Tampere University of \\ Technology, Laboratory of Materials Science, P.O. BOX 589, 33101 Tampere, +358408490145
}

\begin{abstract}
The production of gear components includes numerous manufacturing operations which are carried out to ensure proper surface characteristics of components to deal with wear and fatigue. Surface shot peening is one way to increase the compressive residual stresses on the surface and thus ensure better wear and fatigue resistance. An experimental plan for shot peening was conducted to produce samples with varying surface characteristics. Residual stress profile and Barkhausen noise measurements were carried out for the samples. The objective of the study was to evaluate the interactions between the shot peening parameters studied, the residual stress profiles and the Barkhausen noise measurements. A multivariable regression analysis was applied for the task. Some remarkable correlations were found between the shot peening parameters, residual stress profile and Barkhausen noise features. The most important finding was that when the shot peening intensity was high enough, over $0.5 \mathrm{mmA}$, it dominated the shot peening coverage density parameter and thus no correlations could be gained. On the other hand, if the intensity parameter was lower than the limit of $0.5 \mathrm{mmA}$, the correlation between residual stress and Barkhausen noise measurements was remarkable. This means that the surface Barkhausen noise measurements could be used for the evaluation of the stress gradient in the shot peening process.
\end{abstract}

Keywords: shot peening, Barkhausen noise, fatigue resistance, X-ray diffraction, residual stress

\section{Introduction}

Cost efficiency needs to be as high as possible in the modern metal industry; this means that the produced parts need to be durable enough even though material costs should be low and strong enough for light structures. Shot peening is a cost efficient post treatment and surface finishing process used in the metal industry which can be used for preventing the formation of fatigue failures for example in gears. Fatigue is the most usual cause for gear failures to occur in carburised gears as Shaw et al. [1] explain and it is known that the material surface treatments have a big effect on the durability on the component. Shot peening process is a cold working process where spherical shots are bombarded on to the surface of a treated sample compressing the sample surface structure causing plastic deformation on the surface and subsurface regions and creating compressive stresses. After the shot peening process the surface should have an entirely compressive residual stress state [2]. The produced compressive residual stress gradient can increase the fatigue resistance of the treated part and thus prevent the crack forming from the surface layer, which is important in the case where high wear and fatigue properties 
are demanded. The studies found in the literature consider only the assessment of surface residual stresses. However, it would be beneficial if the residual stress profile could be non-destructively assessed. In this paper, an approach for residual stress profile assessment is presented.

The shot peening process, magnitude and depth of the compressive residual stresses, is controlled by the parameters used which are Almen intensity, coverage and the properties of the shots as concluded by Kostilnik [3]. The challenge is to find a good compromise between shot peening parameters to ensure good quality shot peening. Variables affecting the peening intensity are velocity, hardness, size and weight of the shots and the angle of shots [3]. The shot peening intensity can be measured by Almen intensity [4]. Different intensity values utilised in the shot peening can lead to different depths of the shot peening treatment [5]. Kostilnik [3] describes that the shot peening action may extent up to $0.5 \mathrm{~mm}$ below the surface depending on the parameters. Coverage parameter is the amount of the particles covering the sample surface and can be varied by changing the exposure time of the peening [5]. In addition, the shot size and weight affects into the treatment effect.

There are still some challenges in the process, for example how to find the best parameters for intensity and coverage to get the desired quality of the operation. The Almen intensity procedure does not provide information of the residual stresses on the surface nor the produced residual stress gradient. To verify the quality of the surface layer the produced residual stress profile should be detected on the surface of the samples with non-destructive methods. However, no online nondestructive measurement method for evaluation of the residual stress gradient has been developed yet and thus some indirect method must be applied.

One candidate method for online assessment of residual stress profiles could be magnetic Barkhausen noise (BN) method that is widely used in online detection of grinding burns. The method has also been used in many studies for studying the shot peened samples. The use of the BN method is based on the observation of the irreversible domain wall motion in the ferromagnetic material that produces changes in the magnetization behavior [6]. Many factors affect observed BN signal like grain boundaries [7], dislocations [8], precipitates [8] and also stress state [9]. Barkhausen noise can be utilized in the studies of the shot peened samples because it is sensitive to both stress changes and the microstructural changes. In the research carried out by Zagar et al. [10] the shot peened samples were studied with BN method, X-ray diffraction residual stress depth profiles and hole drilling. Usually, the determination of the residual stress gradient requires material removal. For the material removal without affecting the stresses, the electrochemical polishing method has been found feasible by studies of Alkaisee and Peng [11] to give a fine quality of polished surface with good reproducibility. In the earlier studies, Tiitto and Francino [12] reported already at 1991 that it would be possible to distinguish different types of residual stress profiles from shot peened samples with help of Barkhausen noise. Suominen and Tiitto [13] searched for the BN response from different samples with work hardening, work softening and both due to shot peening actions. They studied the XRD residual stress and FWHM depth profiles and found that the BN classified the work softening and hardening effect with different shot peening intensities.

Savas and Gür [14] studied shot peened sample surfaces by surface X-ray diffraction residual stress measurements and $\mathrm{BN}$ measurements. They noted that in addition to changes in the RMS also the BN peak position showed changes with different shot peening parameters. Kleber and Pirfo Barroso [5] studied the effect of the austenite transforming into martensite from austenite stainless steel samples with BN and X-ray diffraction residual stress depth profiling after the shot peening procedure. Marconi et al. [15] used X-ray diffraction residual stress measurements from shot peened Almen test strips to calibrate the BN method for shot peened sample measurements.

In this study, an experimental design was carried out for providing different levels of coverage density and intensity values for the actual shot peening experiments. Small carburizing gear samples were used for shot peening according to the experimental design. Different methods were utilized for studying the shot peened sample surface and subsurface quality. Barkhausen noise measurements were carried out from the surface and X-ray diffraction method was used for residual 
stress depth profiling. The measured residual stress profiles were characterized by certain features while several typical $\mathrm{BN}$ features were used to represent the $\mathrm{BN}$ measurements. The interactions between shot peening parameters and the features computed were analyzed with multivariable regression analysis. As vast use of different $\mathrm{BN}$ features has not been reported in the earlier works with shot peening samples. The objective of the present study was to link the shot peening parameters studied to the residual stress profiles and $\mathrm{BN}$ measurements and to link the residual stress profiles and $\mathrm{BN}$ measurements.

\section{Materials and methods}

\subsection{Sample preparation and experiments}

The studied samples were small gear samples manufactured from gear steel $17 \mathrm{NiCrMo6}-4$. The initial state for the gear wheels were a carburizing case-hardened and tempered surface before grinding and honing. The case depth was $0.8 \mathrm{~mm}$ and the surface hardness was $61 \mathrm{HRC}$, or $720 \mathrm{HV} 1$. Shot peening was carried out as a final surface treatment. The shot peening parameters which were varied were intensity (obtained by variating the air pressure) and coverage density (obtained by variating the peening time). The peening intensities were measured as per SAE J443 [4] with Almen strips Type A. Intensity and coverage as defined in SAE J2277 [16] are not truly independent parameters as higher intensity, which realized in this study by increasing the speed of the media through higher pressure, will yield bigger impacts, which influences coverage. Therefore, the concept of coverage was replaced in this study by a coverage density expressed in $\mathrm{kg} / \mathrm{cm}^{2}$ defined as:

$$
\text { coverage density }=\frac{\mathrm{CT} * \mathrm{n} * \mathrm{~F}}{\mathrm{~S}},
$$

where $C T$ was the total peening time [s], $n$ was the total number of nozzles (4), $F$ was the shot media flow per nozzle $[6.5 \mathrm{~kg} / \mathrm{s}]$ and $S$ was the area to be peened $\left[220.7 \mathrm{~cm}^{2}\right]$. The varied output parameter in the coverage density was the total peening time. The total peening time was varied between $25.5-76.4$ s. The shot peening media was steel cut wire $0.7 \mathrm{~mm}$ G3 with a hardness of $640 \mathrm{HV} 1$, or $57.5 \mathrm{HRC}$, meaning that the gear samples were harder than the shots used.

To investigate the effect on the shot peening parameters to Barkhausen noise and residual stress outcomes an experiment was conducted with the help of statistical design of experiments. The test plan included six different intensity levels and five coverage density levels. Other shot peening parameters were kept constant. A central composite design for two variables at five levels was developed first (experiments B, D, E, F, G, H, J, K and M in Figure 1). The center point experiment (G) with intensity value of $0.53 \mathrm{mmA}$ and coverage density value of $0.1 \mathrm{~kg} / \mathrm{cm}^{2}$ was repeated five times to estimate the standard deviation involved with the measurements. With this design, it is already possible to analyse nonlinear effects but still four additional experiments were defined for high variable values (experiments A, C, L and N in Figure 1). Also it was desired to test a somewhat higher intensity value so one experiment was added for that (I in Figure 1.). Thus, the total number of experiments was 13 plus the five center point repetitions, giving totally 18 measurements. The total number of different shot peening parameter combinations was 14 . A single shot peening procedure was utilised.

Shot peening parameter test setup is shown in Fig 1. Smaller teeth pieces of samples of the gear were cut for the $\mathrm{X}$-ray diffraction residual stress depth profiling. 


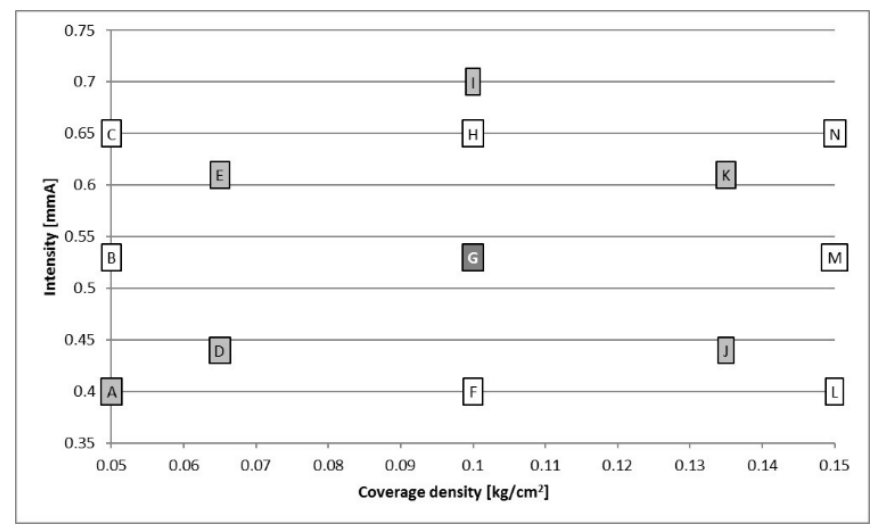

Fig. 1. Shot peening experimental design graph of intensity and coverage density values utilised in tests.

\subsection{Measurements}

Barkhausen noise measurements were made with a Rollscan 300 Barkhausen noise analyser, manufactured by Stresstech Oy (Finland). A commercial gear sensor to fit the gear surface was used in the measurements. Measurements were recorded with MicroScan software, yielding different parameters calculated from the signal itself. Different measurement parameters were utilized: a magnetizing voltage of $5 \mathrm{vpp}$ (voltage peak to peak) was used with magnetizing frequencies of 80 and $200 \mathrm{~Hz}$. In the analyses, the results were an average of 20 Barkhausen noise bursts filtered to 70-200 kHz. In addition, after shot peening, GearScan robot measurements were carried out with the magnetizing voltage of $5 \mathrm{vpp}$ and frequency of $125 \mathrm{~Hz}$.

The residual stresses (RS) and full width at half maximum (FWHM) of the diffraction peak values were examined from the samples using an X-ray diffractometer (XRD) XStress 3000, manufactured by Stresstech Oy (Finland). Residual stress measurements were performed using $\mathrm{CrK} \alpha$ radiation and the modified $\chi(\mathrm{Chi}$ ) method [17]. Residual stresses were derived from measurements on the Fe-211 reflection using the standard $\sin 2 \psi$ method [18] over a total of 10 tilt position within the $45^{\circ}$ to $45^{\circ} \psi$ interval. The current $6.7 \mathrm{~mA}$ and voltage $30 \mathrm{kV}$ was used in the measurements. The collimator size was $2 \mathrm{~mm}$ and the exposure time was $10 \mathrm{~s}$ for the residual stress measurements. The XRD measurements were done in two perpendicular directions being the gear root direction $\left(0^{\circ}\right)$ and perpendicular to this direction $\left(90^{\circ}\right)$. The direction of the $\mathrm{BN}$ measurements corresponds to the tooth flank direction. CrK $\alpha$ radiation gives a measuring depth of $5-6 \mu \mathrm{m}$. This is somewhat less than the information depth of the BN measurements [19].

To determine a residual stress depth profile, material has to be taken away in order to achieve depth resolution. The layer removal was carried out within an electrochemical cell with electrolytic polishing equipment Movipol-3 (Struers) with commercial perchloric acid solution Struers A2. The residual stress measurements were at first carried out at the surface, then electro polishing was used to take the desired layer off and depth was measured with a dial indicator. This routine was carried out with intended depths of $0,5,10,30,50,75,100,150$ and $200 \mu \mathrm{m}$, continuing with $50 \mu \mathrm{m}$ steps until stress value was stabilized.

Shot peening also affects to the retained austenite content of the sample in the surface layer. Thus, retained austenite was measured from the surface and from the same intended depths as the residual stresses.

\subsection{Features describing residual stress profiles}

Two different parameters were obtained from residual stress measurements on each measurement: the stress in MPa and FWHM in degrees, all values in two directions. In this study, the residual stress profile is linked to shot peening parameters and BN features. As such, the residual stress profile information is 
not very usable in numerical analysis and thus some features describing it are computed. These features are obtained by fitting a second order polynomial into the residual stress depth profiles counting depths up to $100 \mu \mathrm{m}$. The equation for the second order polynomial is:

$$
y=a x^{2}+b x+c,
$$

where $a, b$ and $c$ are polynomial coefficients. The fitting of a parabola into the stress profile is illustrated in Figure 2. The features describing the profile were the fitting parameters $a, b$ and $c$ and the location of the parabola peak obtained from the zero of the derivative of (2). This feature is later referred as a parabola peak position. The parabola peak position corresponds to the depth at which the highest compressive stresses occur. In addition, this maximum stress is obtained as one feature describing the stress profile while the surface residual stress is represented by the fitting parameter $c$. The last feature is the area between the parabola and the $\mathrm{x}$-axis. This is later referred as the polynomial area.

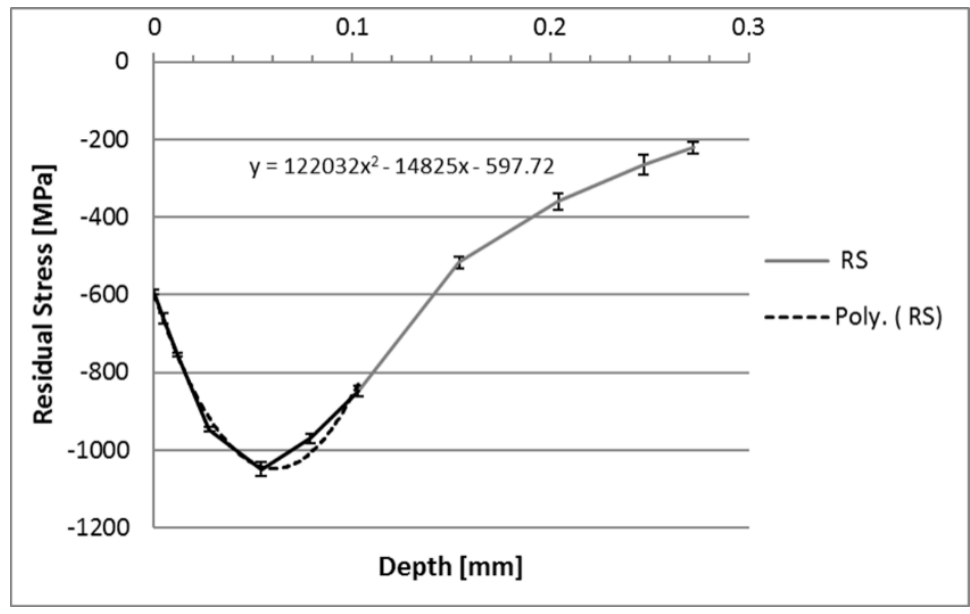

Fig. 2. Example figure on residual stress depth profile in $0^{\circ}$ direction, where $0^{\circ}$ direction means gear root direction. Also shown the fitted parabola up to $100 \mu \mathrm{m}$ and the parabola equation.

\subsection{Features from the $B N$ measurements}

The features describing the properties of the BN signal are readily available from the measuring device. There are altogether 17 features including the root mean square (RMS) value (positive, negative and average), peak value (positive, negative and average), peak position (positive, negative and average), FWHM (positive, negative and average), coercivity, remanence, permeability, integral area and spectrum area. Positive and negative refers to the ascending and descending magnetization fields, respectively, and the average is the average of these. In the ideal situation, the values with ascending and descending fields are exactly the same but in practice they sometimes differ. Figure 3 shows the Barkhausen noise signal before and after the shot peening together with the magnetizing current. When comparing Fig.4 a) and b) it is noticed that the BN activity is smaller in b. The difference is however not that obvious and thus the BN profiles are presented in Fig. 4. The profiles show filtered BN as a function of the magnetizing field. A clear decrease in BN activity (flatter profile) is noticed from Fig.4 after the shot peening. All of these features are taken into account when linking the BN measurements to the residual stress profiles even though it has been shown that some features have high crosscorrelations [19]. 

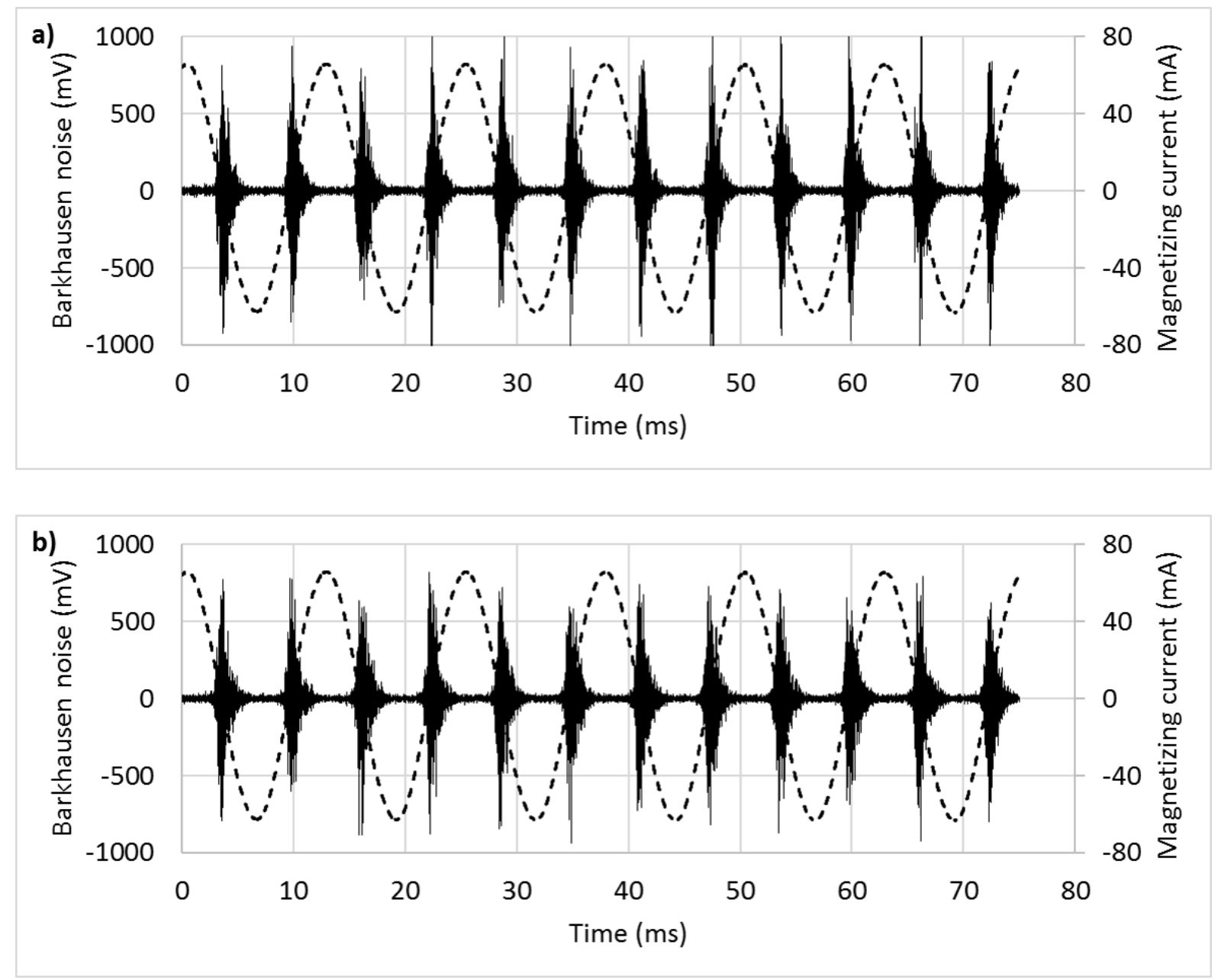

Fig. 3. Barkhausen noise signal (solid line) with magnetizing current (dashed line) as a function of time in a) ascending magnetizing field and $b$ ) descending magnetizing field.

Multivariable regression analysis is a technique used for predicting the unknown value of a variable from the known value of multiple variables. Basically only two types of variables are needed; dependent and independent variables. Dependent variable is predicted in the model and independent variables are used to predict the dependent variable. Multivariable regression analysis is based on a simple equation between $Y$ and $X_{1}, X_{2} \ldots X_{k}$, where $Y$ is the dependent variable and $X_{i}$ are independent variables. The multivariable model in general form is:

$$
Y=b_{0}+b_{1} X_{1}+b_{2} X_{2}+\cdots+b_{k} X_{k},
$$

where $b_{0}$ is the intercept and $b_{1}, b_{2}, \ldots, b_{k}$ are analogous to the slope in linear regression equation and are also called regression coefficients. It should be noticed that an independent variable $X_{i}$ may also be an interaction term or a quadratic or even a higher order term. 


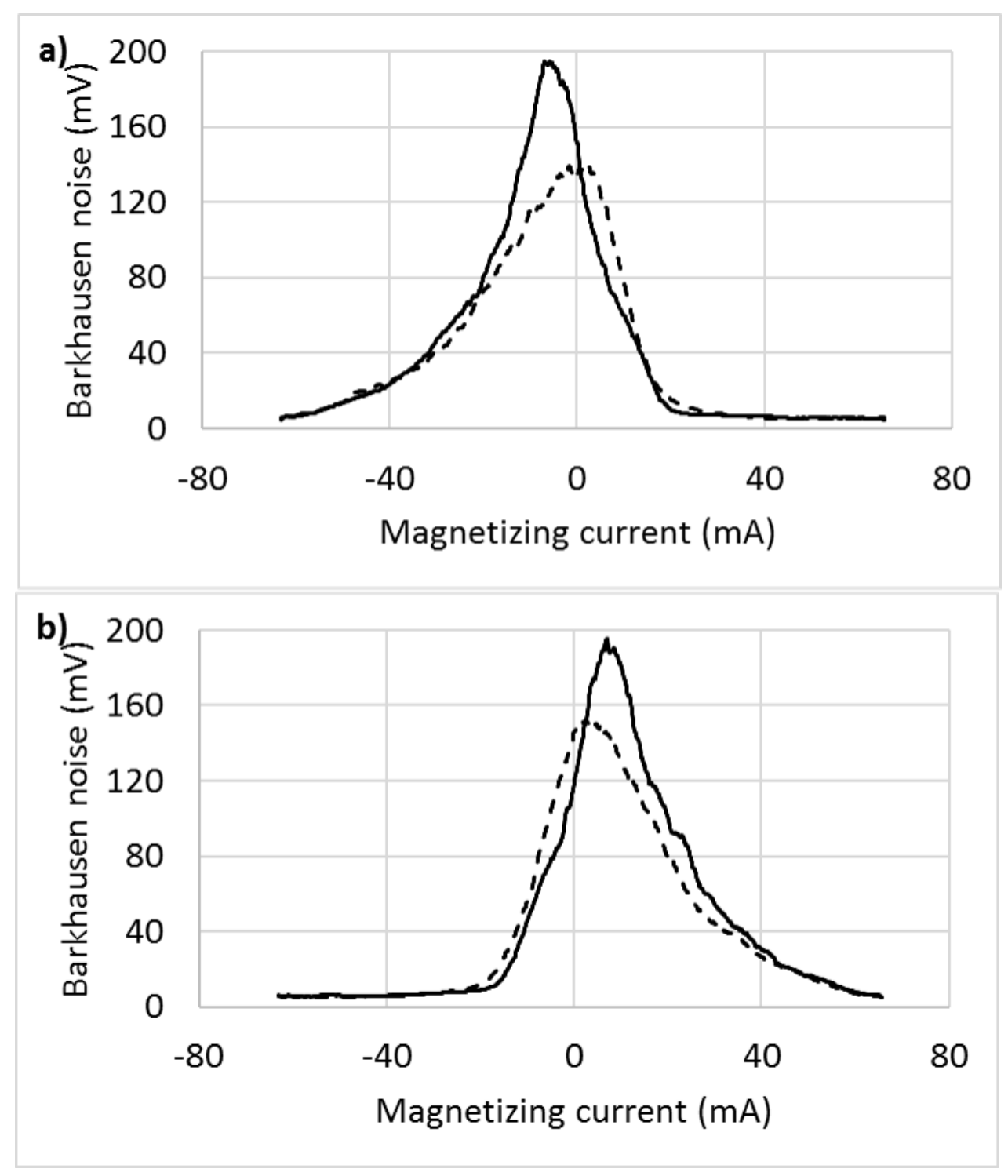

Fig. 4. Barkhausen noise profiles before (solid line) and after (dashed line) the shot peening for a) ascending magnetizing field and $b$ ) descending magnetizing field.

\subsection{Data analysis}

The question is how to select the independent variables for the model. One approach would be to identify a full model with all the possible independent variables and then test each variables' significance with a t-test or an F-test [21]. Another approach would be to form some sort of a ranking for the independent variables and add variables according to the ranking as long as model performance improves [22]. Yet another approach, which also is the approach used in this study, is to use systematic feature selection techniques. Feature selection techniques are numerous and they are not described here in more detail.

The technique adopted here is the so-called floating search algorithm that can be categorised as a deterministic method. The method sequentially uses feature selection, elimination and replacement steps to come up with the optimal feature subset [23]. During each step, candidate models are identified and the most suitable one directs the search towards the optimal solution. The models identified in this study are very simple and actually they only need the forward step of the selection algorithm. The use of the floating search algorithm is justified by the fact that it guarantees that the variable selection does not end up in a local optimum. 
In this study, the number of data points is low and thus the risk for overfitting is evident. To decrease the risk, leave-one-out cross-validation (LOO) is used in model identification. In LOO, $n$ models ( $n$ is the number of data points) are identified. For each model, one data point is left out for model validation while the remaining $n-1$ data points are used for parameter identification. Model performance criteria are calculated based on the validation data points.

The models identified in this study are evaluated through three performance indices. The first one is the coefficient of variation (the $R^{2}$ value) [21]. The $R^{2}$ value tells the fraction of variation that the model in question explains. Thus, the value closer to 1 is better. Too close to 1 is also bad in real-world cases because then the model also estimates the measurement errors. The $R^{2}$ value is given by:

$$
R^{2}=1-\frac{S S_{E}}{S S_{T}}
$$

where $S S_{E}$ and $S S_{T}$ are the residual and total sum of squares, respectively. The second index is the root mean square value of the prediction error (rmse). This index gives indication about the average prediction error. The rmse value is obtained by

$$
\text { rmse }=\sqrt{\frac{1}{n} \sum_{i=1}^{n}\left(y_{i}-\hat{y}_{i}\right)^{2}} .
$$

Above, $y_{i}$ refers to the measured value while $\hat{y}_{i}$ refers to the predicted value. The last index is the average relative error. The relative error for the $i$ :th data point is obtained from:

$$
e_{r, i}=\left|1-\frac{\hat{y}_{i}}{y_{i}}\right| \times 100 \%
$$

\section{Results and discussion}

The section is organized as follows. First, some general remarks from $\mathrm{BN}$ and residual stress measurements are stated. Then the modelling results between shot peening parameters and residual stress profiles are presented followed by the results between residual stress profiles and BN features. Finally the results from models between shot peening parameters and BN features are given.

\subsection{Barkhausen noise results and residual stress profiles}

The Barkhausen noise measurements were carried out from the gear samples before and after the shot peening operation. The overall behavior of the RMS value before and after the shot peening actions on samples can be seen in Figure 5. In addition, Table 1 shows the RMS and standard deviation for centre point test samples with different measurement frequencies. The average value of the RMS calculated from the teeth of one gear sample with $80 \mathrm{~Hz}$ and $200 \mathrm{~Hz}$ measurement frequency showed that in almost all samples the BN RMS value decreased after the different shot peening operations. The measurements taken with $200 \mathrm{~Hz}$ measurement frequency showed even more decreased RMS than the $80 \mathrm{~Hz}$ measurements due to the different measurement depths. It is commonly known that with the smaller measurement frequency, the obtained BN signal comes deeper from the surface layer [24].

Table 1. Average RMS and standard deviation for centre point tests with $80 \mathrm{~Hz}$ and $200 \mathrm{~Hz}$ frequencies before and after shot peening.

\begin{tabular}{lcccc}
\hline & Before SP & After SP & Before SP & After SP \\
\hline Measurement frequency $(\mathrm{Hz})$ & 80 & 80 & 200 & 200 \\
Average RMS & 119.9 & 100.3 & 118.3 & 92.3 \\
Standard deviation & 12.0 & 10.7 & 15.8 & 8.8 \\
\hline
\end{tabular}




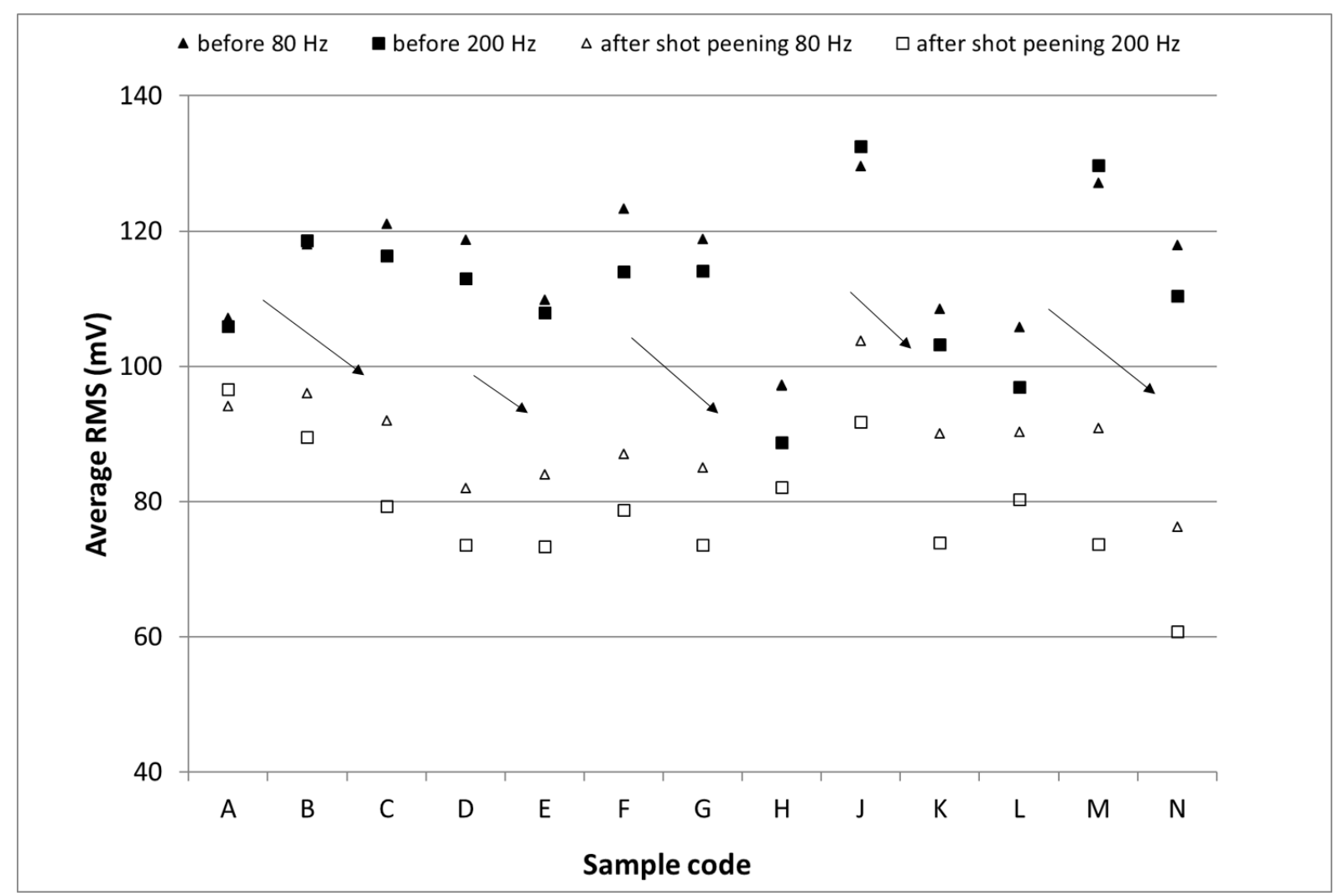

Fig. 5. The Barkhausen noise average RMS values of teeth of gear samples before and after shot peening with frequencies $80 \mathrm{~Hz}$ and $200 \mathrm{~Hz}$. Sample code in $\mathrm{x}$-axis is arranged according to increasing coverage density and arrows show the increasing intensity.

The curve of the residual stress depth profile presented in Figure 2 suggests a trend by which the residual stresses present at the surface will increase with depth, reaching a peak compressive residual stresses normally at $0.05 \mathrm{~mm}$ under the surface, and then proceed to decrease as the distance from the surface increases. The same trend was observed in all 14 separate samples measured. It was also discovered that the austenite amount in these samples were not that important when correlating the shot peening parameters into the residual stresses and the Barkhausen noise measurements. The original retained austenite volume fraction percent in the honed surface was measured to be $9.4 \%$ with standard deviation of $1.2 \%$.

\subsection{Interaction between shot peening parameters and residual stress profiles}

The models for analyzing the interactions between shot peening parameters and residual stress profiles were identified as described in Section 3.6. The model where shot peening intensity was calculated based on all the stress profile parameters reduced to a model where only the depth of the highest compressive stress (i.e. zero of the derivative $(x)$ ) was used. The $R^{2}$ value for this model was 0.8 showing that a strong correlation exists. Figure 6 shows the intensity values as a function of the predicted intensity values calculated with the model. The input variable for the model was the parabola peak position. Thus, it is evident that shot peening intensity mainly affects the depth of the highest compressive stress. This finding is nicely in accordance with the literature as Suominen and Tiitto [13] and Kleber and Pirfo Barroso [5] stated that treatment depth of shot peening varies depending on intensity. No significant relationship between other stress profile features and intensity was found. While it was shown in [14] that increased intensity also increases compressive residual stresses.

When analysing the results from shot peening coverage density and the residual stress profiles, it was found out that when shot peening intensity is too high, no correlation can be found (as 
seen in Figure $7, \mathrm{R}^{2}=0.0001$ ), but when intensity was below $0.5 \mathrm{mmA}$ then the correlation between coverage density and residual stress was remarkable. Figure 7 shows the surface residual stress correlation with coverage density. The samples were divided into groups: intensity below $0.5 \mathrm{mmA}$ and above $0.5 \mathrm{mmA}$ in Figure 7. The modelling showed that a relationship exists between the polynomial area and coverage density. The $\mathrm{R}^{2}$ value was 0.93 . That relationship is shown in Figure 8 , which shows the predicted coverage density as a function of the measured coverage density for those samples with intensity below $0.5 \mathrm{mmA}$. The polynomial area indicates the average residual stress of the profile. Thus, the conclusion is that increasing coverage density also increases compressive stresses but that effect is only observable when intensity is low enough (below $0.5 \mathrm{mmA}$ ). Coverage can be manipulated by the exposure time (peening time) as suggested by Kleber and Pirfo Barroso [5]. Thus, a similar finding was reported in Zagar et al. [10] where longer exposure time (i.e. higher coverage) led to higher compressive stresses.

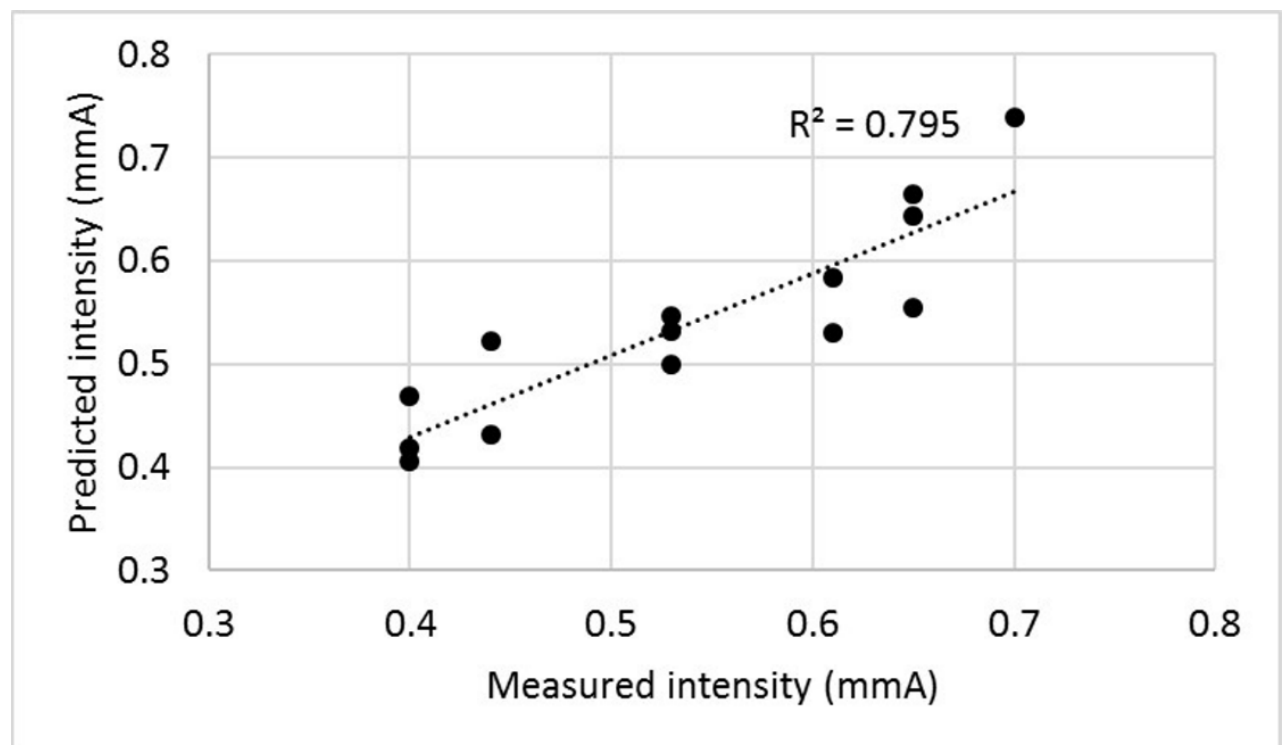

Fig. 6. Predicted intensity as a function of the measured intensity calculated with the model. The input variable for the model was the parabola peak position.

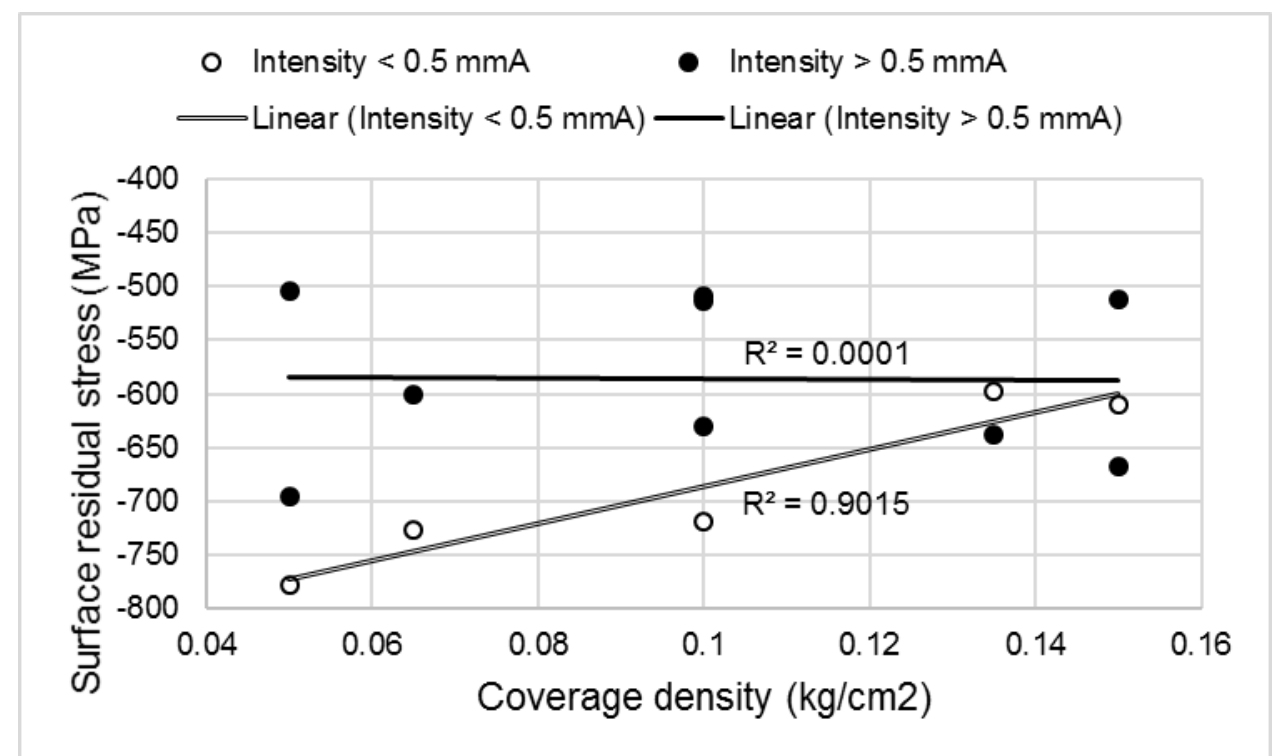

Fig. 7. Surface residual stress correlation with coverage density. The samples were divided into groups intensity below $0.5 \mathrm{mmA}$ and above $0.5 \mathrm{mmA}$ in Figure 7 


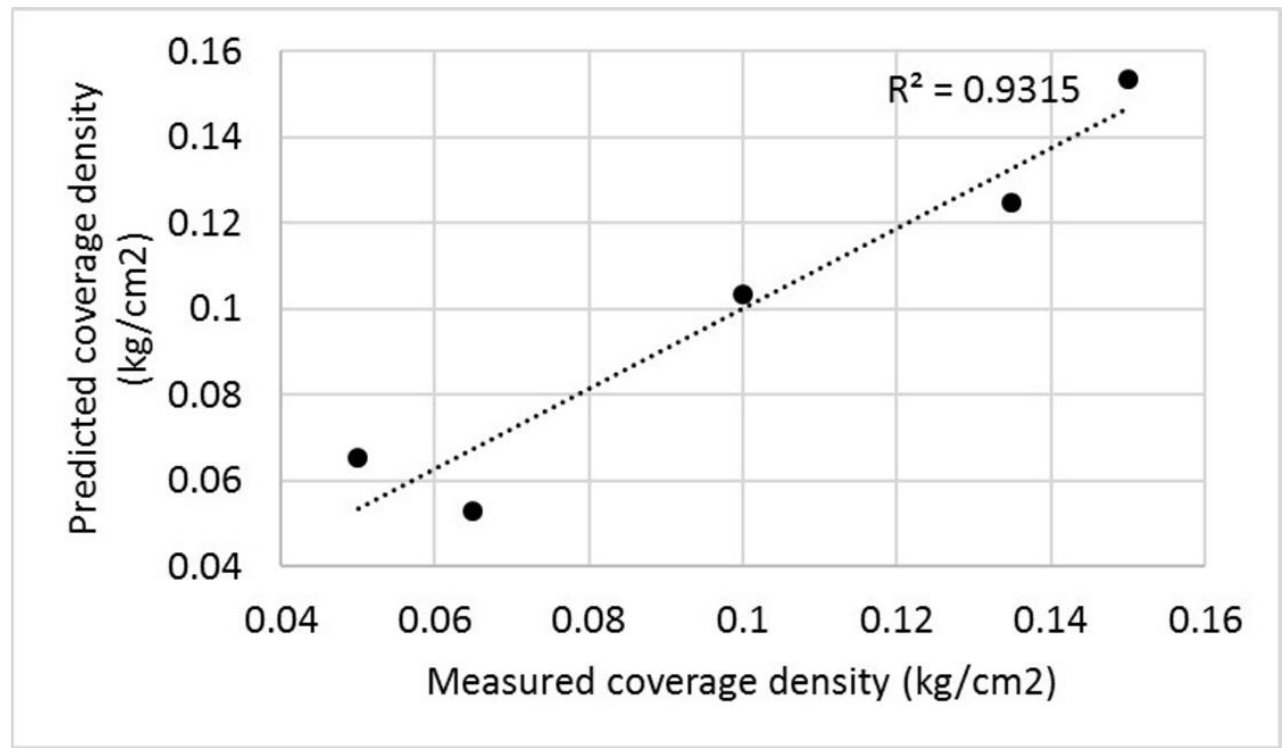

Fig. 8. Coverage density as a function of polynomial area calculated with the model with polynomial area as the input variable.

\subsection{Interaction between residual stress profiles and $\mathrm{BN}$ features}

Models were identified to evaluate the interactions between residual stress profiles and BN measurements. As written earlier there are five features describing the residual stress profile and 17 features describing the BN measurement. Models for each feature describing residual stress profiles were identified as described in 3.5. Significant relationships were found between the BN features and the parabola peak position (Figure 9 ). The $\mathrm{R}^{2}$ value was 0.68 . The model links the parabola peak position to $\mathrm{BN}$ peak position, spectrum area and permeability. Alone these three $\mathrm{BN}$ features are weak $\left(\mathrm{R}^{2}<\right.$ 0.25 ) but together they form a combination that can be used to predict the parabola peak position with the reasonable accuracy $\left(\mathrm{R}^{2}=0,681\right)$. Barkhausen noise peak position is usually related to hardness in the literature $[25,26]$. However, it has also been found to be significant in the prediction of residual stress [20]. As mentioned earlier, shot peening intensity affects the residual stress state and obviously, it affects also to surface hardness. Thus, the changes in BN peak position reflects the changes in both of these. Permeability can be linked to the microstructural features, to domain wall motion and its greatest degree of freedom to move [27] and thus its selection can be justified. 


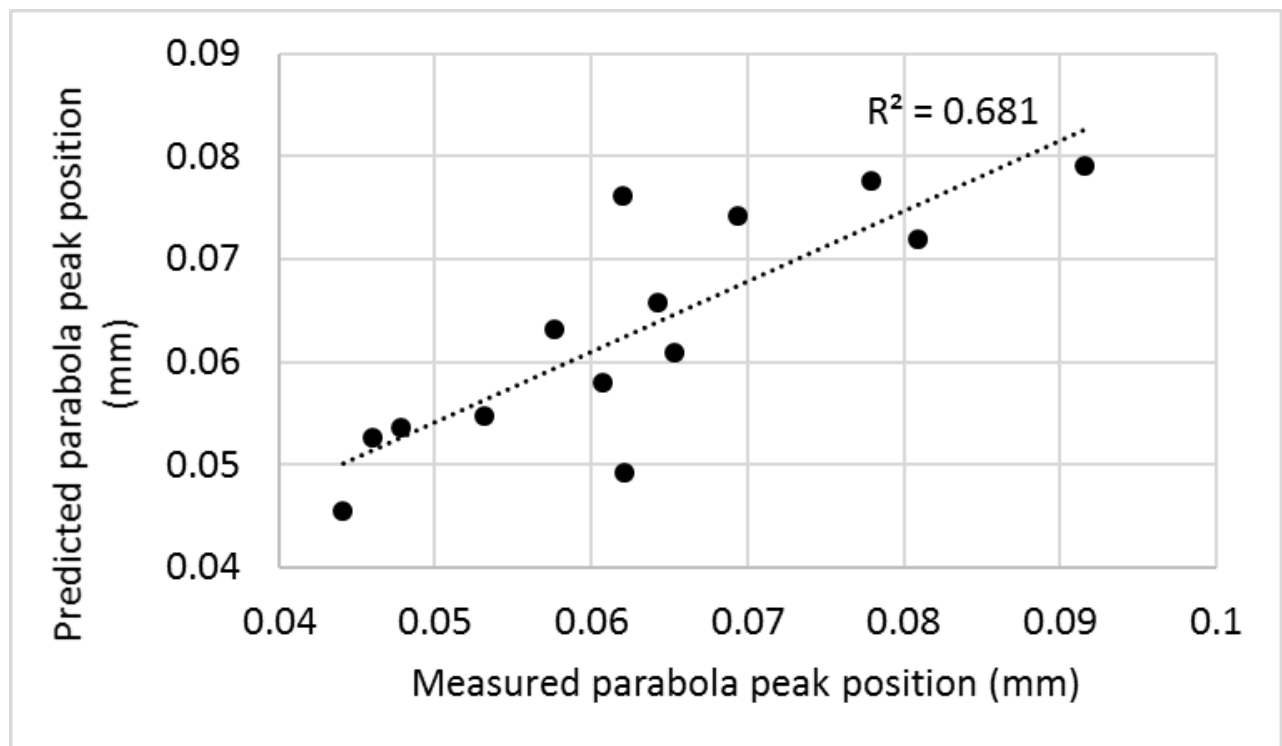

Fig. 9. Parabola peak position as a function of their measured values calculated with the model. The input variables of the models are $\mathrm{BN}$ peak position, spectrum area and permeability.

The BN parameter peak width is related with the polynomial area of the residual stress profile (Figure 10) which is further linked to coverage density (as show in Figure 7 ). The $R^{2}$ value was 0.89 . As mentioned earlier, higher coverage basically means longer peening time. As the peening time increases also the material work hardens more. Peak width value has been reported increasing with higher hardness and thus its selection is justified. In addition, the surface residual stress depends on Barkhausen noise features coercivity and permeability as shown in Figure 11 . The $\mathrm{R}^{2}$ value was 0.55 .

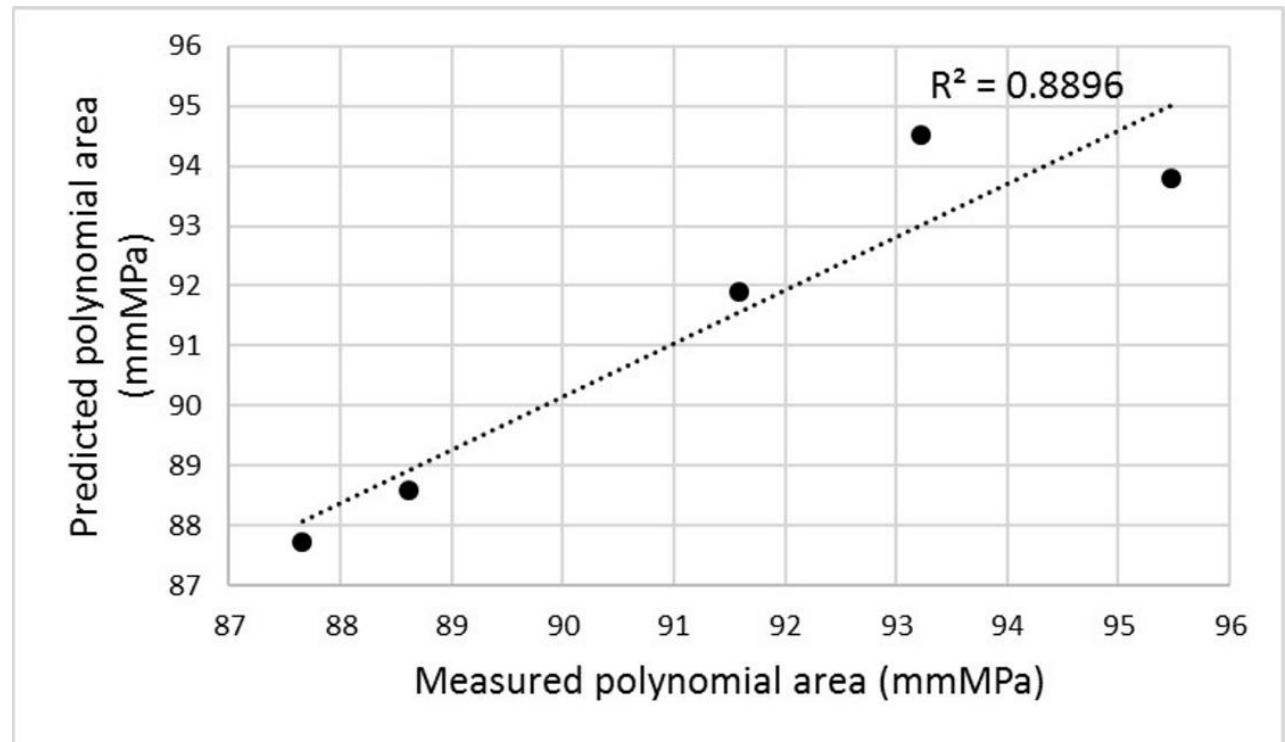

Fig. 10. Polynomial area as a function of their measured values calculated with the model. The input variable of the model is $\mathrm{BN}$ peak width. 


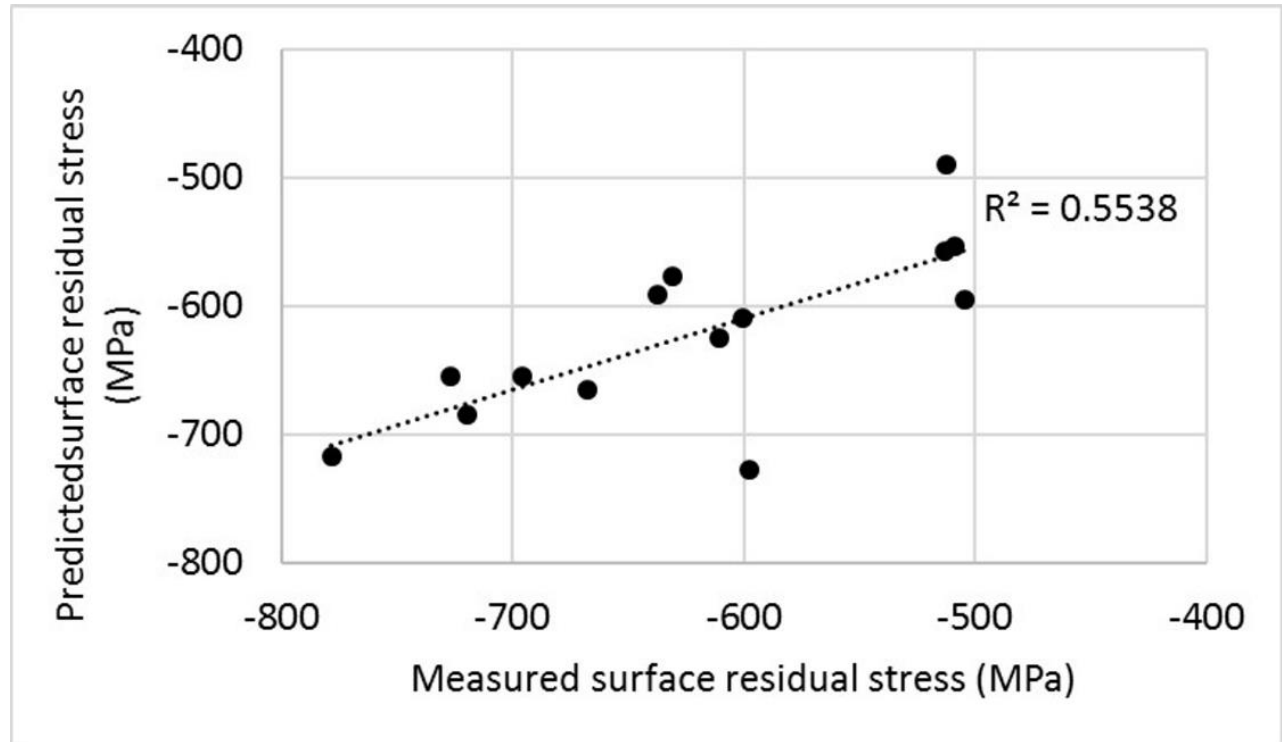

Fig. 11. Surface residual stress as a function of their measured values calculated with the model. The input variables of the model are coercivity and permeability.

\subsection{Interaction between shot peening parameters and $\mathrm{BN}$ features}

Figure 12 shows the intensity as a function of their measured values. The $\mathrm{R}^{2}$ value was 0.8 . Model input variables were $\mathrm{BN}$ peak position, remanence and permeability. Figure 13 shows the coverage density as a function of their measured values. The $\mathrm{R}^{2}$ value was 0.57 . Model input variables were peak width and spectrum area.

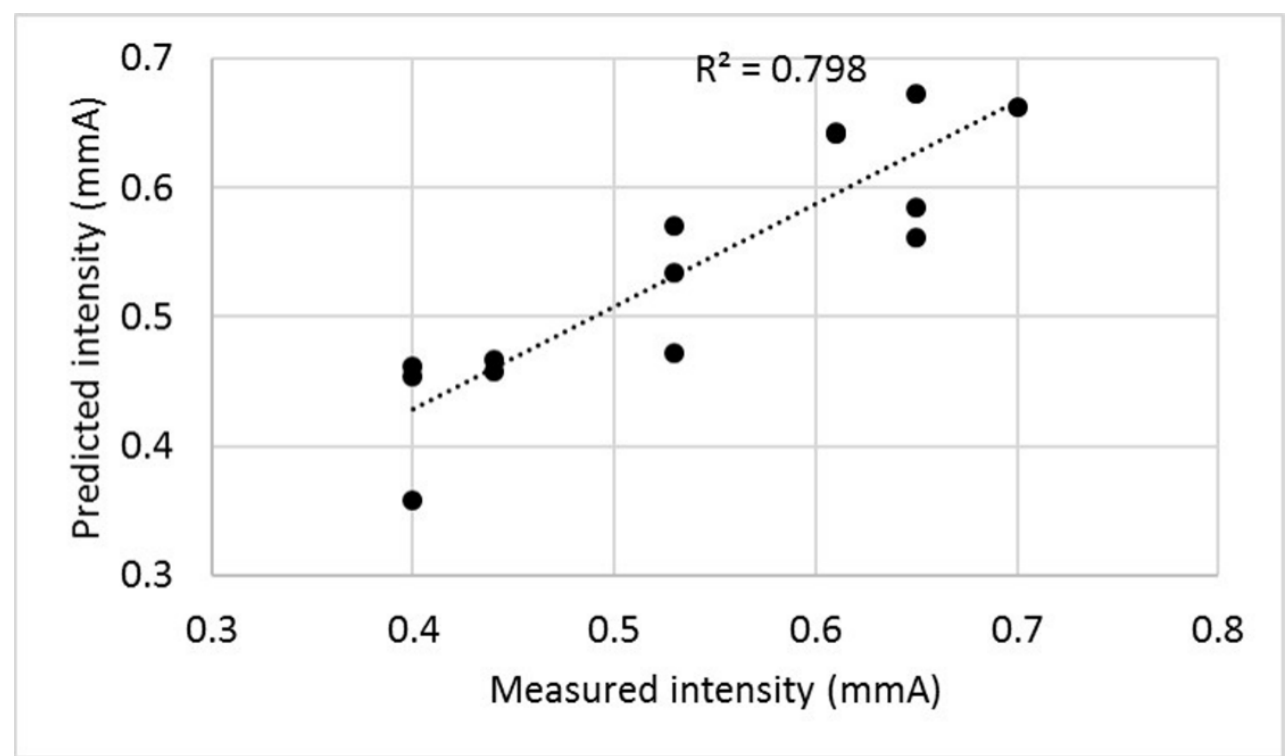

Fig. 12. Intensity as a function of their measured values calculated with the model. The input variables of the model are BN peak position, remanence and permeability. 


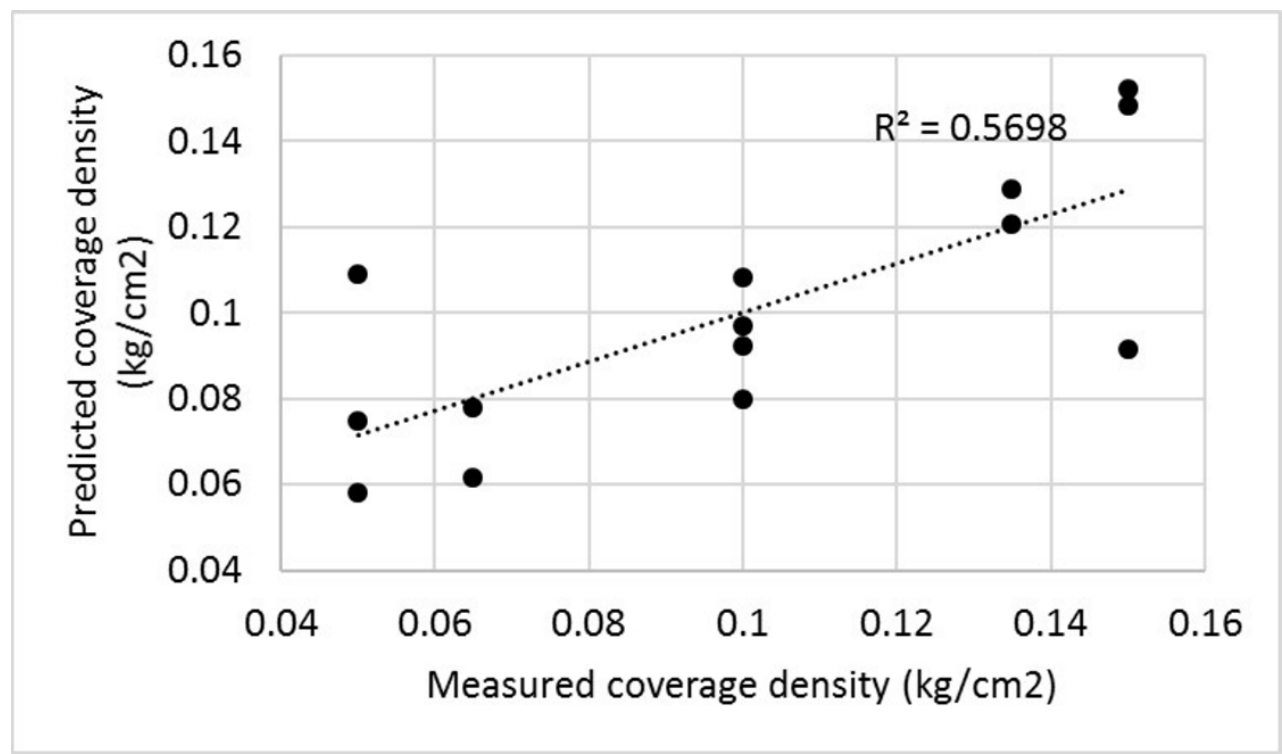

Fig.13. Coverage density as a function of their measured values calculated with the model. The input variables of the model are peak width and spectrum area.

\subsection{Collection of the results}

Table 2 collects the performance indices of the identified models. In general, the models perform well. The lowest $R^{2}$ value is obtained for the surface residual stress vs. BN features relationship. The table also gives the relative errors of the models. It is noticed that standard errors are rather low when compared with the ranges of the modelled variables. As mentioned in the introduction, non-destructive evaluation of the residual stress profile is significant. The results show that the correlations between the residual stress profile and $\mathrm{BN}$ features are found even though more work is needed. The other relationships found can be used in verification of the shot peening results and the models identified. If shot peening is carried out with certain parameter values producing a certain residual stress profile, the correlations between shot peening parameters and $\mathrm{BN}$ features should be similar to those found in this study.

Table 2. Collection of results.

\begin{tabular}{llllll}
\hline Relationship & Figures & Input variable & $R^{2}$ & rmse & Relative error \\
\hline $\begin{array}{l}\text { Shot peening parameters vs. residual } \\
\text { stress profile }\end{array}$ & & & & & \\
$\quad$ Intensity vs. RS profile & 6 & parabola PP & 0.80 & 0.047 & $6.7 \%$ \\
$\quad$ Coverage density vs. RS profile & 7 & pol. area & 0.93 & 0.010 & $12.5 \%$ \\
RS profiles vs. BN features & & & & & \\
$\quad$ Parabola peak position vs.BN features & 9 & BN PP, sp. area, perm & 0.68 & 0.007 & $9.4 \%$ \\
$\quad$ Polynomial area vs. BN features & 10 & peak width & 0.89 & 0.96 & $0.7 \%$ \\
$\quad$ Surface RS vs. BN features & 11 & coer, perm, & 0.55 & 57.7 & $7.9 \%$ \\
Shot peening parameters vs. BN features & & & & & \\
$\quad$ Intensity vs. BN features & 12 & BN PP, rem, perm & 0.80 & 0.047 & $8.0 \%$ \\
$\quad$ Coverage density vs. BN features & 13 & BN PW, sp. area & 0.57 & 0.025 & $21.8 \%$ \\
\hline
\end{tabular}

The coverage density and intensity values of the shot peening process were the only variables studied in this paper. Other shot peening variables were kept constant. Totally 17 features from the BN signal and five features describing the residual stress profile were used in the studies. As wide use of different Barkhausen noise parameters were not found in the earlier studies. Poorna Chandler et al. [28] used the RMS value and peak height to correlate the shot peened variables with the Barkhausen noise response. They [28] noticed that the blasting pressure and the impingement angles have significant effects on $\mathrm{BN}$ parameters while the effect of the used grit size in peening was 
statistically insignificant. The studies of Marconi et al. [15] have correlated only the RMS value to the shot peened variables and they noticed an increasing trend of RMS value after the shot peening operation. This was explained as the peening procedure transformed the residual austenite into martensite and this affected the magnetic behaviour of the material [15]. Savas and Gür [14] explained that the decrease in the MBN emission with increasing shot peening intensity can be explained by the negative effect of compressive residual stresses on the motion capability of $180^{\circ}$ domain walls. The same observation was made in this study. Wojtas [29] concluded that in relatively soft steels the BN level decreases with increasing shot peening intensity while in contrast in very hard martensitic materials it increases. Wojtas [29] justified the behavior so that in the soft steels this effect reflects the growing compressive stresses and work hardening during shot peening and in the harder materials the microstructural changes and work softening occurs in the very near surface layers of the material [29].

\subsection{Future work}

To improve the analysis of the shot peening process, one idea would be to make a new set of samples with different test setup and more samples with less than $0.5 \mathrm{mmA}$ intensity parameter to verify the results gained from this study. It is also worthwhile to test different shot peening materials also with the method to verify the operation on other materials. In addition, fatigue tests from the differently shot peened gear samples would provide information of the fatigue behaviour of the samples. The utilization of magnetic Barkhausen noise as a tool for online non-destructive measurement method for evaluation of the residual stress gradient would give huge potential for the industrial users i.e. shot peening users. However, the implementation of the measurement process would need much more work with different materials and shot peening parameters. More samples are needed to verify the results more widely before considering the method as a commercially available inspection method.

\section{Conclusions}

In this study, a shot peened gear sample series was studied with Barkhausen noise and X-ray diffraction based residual stress measurements. Based on the measurements some remarkable correlations were found between the shot peening parameters, residual stress depth profile and Barkhausen noise measurements. This means that the surface Barkhausen noise measurements could be used for evaluation of the stress profile produced in shot peening process. The shot peening intensity was found to be dominating if the parameter value was above $0.5 \mathrm{mmA}$. Then a relationship was found between intensity and parabola peak position, which is associated with depth where the highest compressive stress occurs. If the intensity parameter was under the $0.5 \mathrm{mmA}$ limit, the Barkhausen noise and the residual stress measurements correlated well with the changes in coverage density. Reasonable correlations were also found between BN features and residual stress profiles. After more testing with different materials and shot peening parameters, the method that utilises the combined features from the BN signal could be proposed as an online measurement tool for evaluating the residual stress gradients non-destructively.

\section{Acknowledgments}

Support from Foundation of Emil Aaltonen, Stresstech group and Academy of Finland is gratefully acknowledged.

\section{References}

1. Shaw BA, Ashby R, Evans JT (2000) The Use of Controlled Post Heat Treatment Cleaning and Peening to Enhance the Fatigue Strength and Reliability of Case Carburized Gears.

DOI:10.1361/cp2000ht0046 
2. Canale FLC, Totten GWE, Mesquita GA (2008) Failure Analysis of Heat Treated Steel Components, ASM International

3. Kostilnik T (1994) Surface Engineering: Shot Peening. In: ASM Handbook, ASM International, 5:126-135

4. SAE Standard: J443- Procedures for Using Standard Shot Peening Test Strip (2003)

5. Kleber X, Pirfo Barroso S (2010) Investigation of shot-peened austenitic stainless steel 304L by means of magnetic Barkhausen noise. Mater Sci Eng A. DOI:10.1016/j.msea.2010.06.008

6. Chikazumi S (1964) Physics of Magnetism. John Wiley \& Sons, Inc.

7. Jiles DC (2000) Dynamics of domain magnetization and the Barkhausen effect. Czechoslov J Phys. 50:893-988

8. Jiles DC (1991) Introduction to Magnetism and Magnetic Materials. Chapman and Hall

9. Cullity BD (1972) Introduction to Magnetic Materials. Addison-Wesley Publishing Company

10. Žagar S, Žerovnik P, Grum J (2009) Surface layer analysis after shot peening by Barkhausen noise signal. In: The $10^{\text {th }}$ International Conference of the Slovenian Society for Non-destructive Testing, Application of Contemporary Non-Destructive Testing in Engineering, (Ljubljana) pp 539-548.

11. Alkaisee R, Peng RL (2014) Influence of Layer Removal Methods in Residual Stress Profiling of a Shot Peened Steel Using X-Ray Diffraction. Adv Mater Res.

DOI:10.4028/www.scientific.net/AMR.996.175

12. Tiitto K, Francino P (1991) Testing shot peening stresses in the field. The Shot Peener, 4(4):1-2

13. Suominen L, Tiitto K (1993) Evaluation of quality in shot peened components with Barkhausen noise. In: Ericson T, Pluvinage G, Castex L (ed) MAT-TEC 93: Improvement of Materials, Detroit, pp 271-277

14. Savaş S, Gür CH (2010) Monitoring variation of surface residual stresses in shot peened steel components by the magnetic Barkhausen noise method. Insight. DOI:10.1784/insi.2010.52.12.672

15. Marconi P, Lauro M, Bozzolo W (2003) Shot Peening on Pelton Wheels: Methods of Control and Results. In: 4th International Conference on Barkhausen Noise and Micromagnetic Testing, Italy, pp $151-158$

16. SAE Standard: J2277- Shot Peening Coverage Determination (2013)

17. SFS Standard: (2008) SFS-EN15305, Non-destructive Testing- Test Method for Residual Stress Analysis by X-ray Diffraction

18. Noyan IC, Cohen JB (1987) Residual Stress Measurement by Diffraction and Interpretation. Springer-Verlag, New York. DOI: 10.1007/978-1-4613-9570-6

19. Hauk V (1997) Structural and Residual Stress Analysis by Nondestructive Methods. Elsevier, Netherlands. DOI:10.1016/B978-044482476-9/50001-3.

20. Sorsa A et al (2012) Quantitative prediction of residual stress and hardness in case-hardened steel based on the Barkhausen noise measurement. NDT E Int. DOI:10.1016/j.ndteint.2011.11.008

21. Hays WL (1994) Statistics, $5^{\text {th }}$ ed., Holt, Rinehart and Winston, Inc. 
22. Guyon I, Elisseeff A (2003) An introduction to Variable and Feature selection. J Mach Learn Res. 3:1157-1182

23. Nakarivakul S, Casacent DP (2009) An improvement on floating search algorithms for feature subset selection. Pattern Recogn Lett. DOI:10.1016/j.patcog.2008.11.018

24. Moorthy V, Shaw BA, Evans JT (2003) Evaluation of tempering induced changes in the hardness profile of case-carburised EN36 steel using magnetic Barkhausen noise analysis. NDT E Int.

DOI:10.1016/S0963-8695(02)00070-1

25. Davut K, Gür CH (2007) Monitoring the microstructural changes during tempering of quenched SAE 5140 steel by Magnetic Barkhausen noise. J Nondestruct Eval. DOI:10.1007/s10921-007-0025-x

26. Stewart DM, Stevens KL, Kaiser AB (2004) Magnetic Barkhausen noise analysis of stress in steel. Curr Appl Phys. DOI:10.1016/j.cap.2003.11.035

27. Karimian N et al (2014) Differential permeability behaviour of P9 and T22 power station Steels. J Magn Magn Mater. DOI:10.1016/j.jmmm.2013.09.059.

28. Poorna Chander K et al (2009) Effects of grit blasting on surface properties of steel substrates. Mater Des. DOI:10.1016/j.matdes.2009.01.014

29. Wojtas A (2004) Surface and subsurface residual stresses after shot peening- their qualitative and quantitative analysis by x-ray diffraction and Barkhausen noise analysis. Met Finish News. 5:24-26 